\title{
Systems Analysis and Improvement Approach to Optimize the Hypertension Diagnosis and Case Cascade for PLHIV Individuals (SAIA-HTN): A Hybrid Type III Cluster Randomized Trial
}

Sarah Gimbel ( $\nabla$ sgimbel@uw.edu )

University of Washington https://orcid.org/0000-0003-3330-1208

Ana Olga Mocumbi

Universidade Eduardo Mondlane Faculdade de Medicina

Kristjana Ásbjörnsdóttir

University of Washington

Joana Coutinho

Health Alliance International

Leonel Andela

Ministry of Health Mozambique

Bonifacio Cebola

Ministry of Health Mozambique

Heidi Crane

University of Washington

Jonny Crocker

University of Washington

Leecreesha Hicks

Health Alliance International

Sarah Holte

University of Washington

Rodrigues Hossieke

Ministry of Health Mozambique

Edgar Itai

Ministry of Health Mozambique

Carol Levin

University of Washington

Nelia Manaca

Health Alliance International

Filipe Murgorgo

Ministry of Health Mozambique 


\section{Miguel Nhumba}

Health Alliance International

\section{James Pfeiffer}

University of Washington

\section{Isaias Ramiro}

Health Alliance International

\section{Keshet Ronen}

University of Washington

\section{Nona Sotoodehnia}

University of Washington

\section{Onei Uetela}

University of Washington

\section{Anjuli Wagner}

University of Washington

\section{Bryan Weiner}

University of Washington

\section{Kenneth Sherr}

University of Washington

\section{Study protocol}

Keywords: Systems analysis and improvement approach (SAIA), Hypertension, CFIR, ORIC, process mapping, cascade analysis, continuous quality improvement, implementation science, systems engineering, HIV

Posted Date: February 11th, 2020

DOl: https://doi.org/10.21203/rs.2.23120/v1

License: (c) (i) This work is licensed under a Creative Commons Attribution 4.0 International License. Read Full License

Version of Record: A version of this preprint was published at Implementation Science on March 6th, 2020. See the published version at https://doi.org/10.1186/s13012-020-0973-4. 


\section{Abstract}

\section{Background}

Across sub-Saharan Africa, evidence-based clinical guidelines to screen and manage hypertension exist; however, country level application is low due to lack of service readiness, uneven health worker motivation, weak accountability of health worker performance, and poor integration of hypertension screening and management with chronic care services.

The Systems Analysis and Improvement Approach (SAIA) is an evidence-based implementation strategy that combines systems engineering tools into a five-step, facility-level package to improve understanding of gaps (cascade analysis), guide identification and prioritization of low-cost workflow modifications (process mapping), and iteratively test and redesign these modifications (continuous quality improvement). As hypertension screening and management are integrated into chronic care services in sub-Saharan Africa, an opportunity exists to test whether SAIA interventions shown to be effective in improving efficiency and coverage of HIV services can be effective when applied to the noncommunicable disease services that leverage the same platform. We hypothesize that SAIA-Hypertension (SAIA-HTN) will be effective as an adaptable, scalable model for broad implementation.

\section{Methods}

We will deploy a Hybrid Type III cluster randomized trial to evaluate the impact of SAIA-HTN on hypertension management in eight intervention and eight control facilities in central Mozambique. Effectiveness outcomes include hypertension cascade flow measures (screening, diagnosis, management, control), as well as hypertension and HIV clinical outcomes among people living with HIV. Cost-effectiveness will be estimated as the incremental costs per additional patient passing through the hypertension cascade steps and the cost per additional disability-adjusted life year averted, from the payer perspective (Ministry of Health). SAIA-HTN implementation fidelity will be measured, and the Consolidated Framework for Implementation Research will guide qualitative evaluation of the implementation process in high- and low-performing facilities to identify determinants of intervention success and failure, and define core and adaptable components of the SAIA-HTN intervention. The Organizational Readiness for Implementing Change scale will measure facility-level readiness for adopting SAIA-HTN.

\section{Discussion:}

SAIA packages user-friendly systems engineering tools to guide decision-making by front-line health workers to identify low-cost, contextually appropriate chronic care improvement strategies. By integrating SAIA into routine hypertension screening and management structures, this pragmatic trial is designed to test a model for national scale-up.

\section{Background}


Hypertension is the leading risk factor for death globally and is more prevalent in sub-Saharan Africa (sSA).[1] A systematic review across the region reported hypertension prevalence between $15-70 \%$ (median prevalence 29\%).[2] Yet most hypertension in SSA is undiagnosed and untreated, further worsening the region's rising burden of cardiovascular disease.[3-5] In Mozambique (a low-income country with $>13 \%$ adult HIV prevalence) [6] hypertension prevalence among adults 25-64 years increased significantly from 2005 to 2015 , now affecting nearly $40 \%$ of adults.[7]

HIV and hypertension comorbidity is common and increasing, in large part due to an aging PLHIV population and side effects of antiretroviral therapy (ART). Survivorship amongst people living with HIV (PLHIV) has increased due to improvements in ART access and effectiveness, thus expanding the number of older PLHIV, who are more likely to experience comorbid hypertension.[8-12] The prevalence of hypertension in PLHIV is estimated to range from 9\%-46\% in LMICs,[13, 14] similar to high income countries.[15-17] Patients on ART are more likely to have dysglycemia, hypertriglyceridemia, and lower levels of high-density lipoprotein cholesterol,[18-20] which may increase hypertension risk. Also relevant, studies indicate that HIV may increase the risk of non-communicable disease (NCD), including CVD, due to stimulation of inflammatory markers and adverse events of ART.[21-24]

Across sSA (including Mozambique), efficacious, low-cost, and safe HTN screening and treatment options exist, and evidence-based clinical guidelines for hypertension management have been developed and disseminated by the Pan-African Society of Cardiology (PASCAR) that recommend routine screening and management of adult PLHIV for CVD risk factors.[25] Yet guideline application is low due to a lack of service readiness (e.g. availability of essential equipment, health worker training), uneven health worker motivation, weak accountability of health worker performance, and poor integration of hypertension screening and management within chronic care services.[26] As a result, self-reported knowledge of blood pressure status and treatment coverage is low. Less than half of individuals with hypertension in sSA are aware of their condition.[27] In Mozambique, less than $15 \%$ of hypertensive adults are aware of their hypertension status (the lowest in SSA); among these, only $50 \%$ are in treatment, less than half of whom have controlled hypertension.[7] As a result of this leaky hypertension cascade, only $3 \%$ of the total adult population with hypertension in Mozambique have their condition controlled.

Approaches to optimize care cascades are needed to maximize the benefits of hypertension screening and management for PLHIV.[28] Barriers to hypertension diagnosis and management for PLHIV exist on the individual (patient and/or caregiver),[26, 29] interpersonal (provider),[30, 31] health systems,[32] and policy levels.[33] Existing research emphasizes individual and interpersonal-level barriers; however, health systems barriers receive less attention,[34] presenting an opportunity to improve patient health by optimizing the complex hypertension cascade. The hypertension cascade presents a series of linked steps (screening, diagnosis, management and control), whereby achieving improved patient outcomes is conditional on successful progression through all service steps. Robust systems engineering methods (e.g. cascade analysis, process mapping, and continuous quality improvement) can optimize poorly functioning cascades by 1 ) identifying main drivers for system inefficiency, 2) supporting locally informed provider decision-making to prioritize intervention, and 3) improving integration of services to 
meet patient needs across diverse chronic care contexts.[28, 35] Low-cost, systems-level interventions are effective and efficient approaches to improve linked cascade services, and may be effective for routinizing hypertension diagnosis and management within existing ambulatory services; addressing both individual and system-level barriers; improving flow through the hypertension care cascade; and ultimately improving patient-level outcomes.

The Systems Analysis and Improvement Approach (SAIA) is designed to optimize cascade performance, is feasible for frontline healthcare workers and managers and may be applicable to optimize the hypertension testing and treatment cascade for people living with HIV (PLHIV) across multiple contexts. SAIA, an evidence-based strategy, which is flexible to local context, supports frontline facility staff to gain a comprehensive view of their complex delivery system, identify and prioritize areas to improve, and iteratively test modifications to increase system outputs and patient outcomes.[36, 37] Health workers in the original cluster randomized trial that tested the intervention to improve prevention of mother-to-child HIV transmission (PMTCT) services noted that SAIA stimulated communication, consensus decisionmaking, and accountability across multiple service points in their facility (e.g. patient care, pharmacy, laboratory services); was accessible by relying on routinely collected data to guide decision-making in a real-world service delivery environment; and resulted in significant improvements in service delivery outcomes.[37] SAIA for the hypertension cascade for PLHIV (SAIA-HTN), was successfully piloted in outpatient services in the study area over a 3-month period, and demonstrated a $31 \%$ increase in hypertension screening.[38] Given the current health service delivery challenges and the promising pliot results, ascertaining whether SAIA-HTN is an effective, adaptable, and scalable model for broad implementation is scientifically important.

\section{Goals and objectives}

The overall goal of this study is to evaluate a model for systematic assessment and improvement of hypertension diagnosis and management services for PLHIV individuals in Mozambique (SAIA-HTN). Over the five-year project, the investigators will conduct a facility-level parallel hybrid type III cluster randomized trial[39] to evaluate SAIA-HTN effectiveness on hypertension cascade optimization (Aim 1); identify drivers of implementation success (Aim 2); and determine costs and cost effectiveness on hypertension and HIV clinical measures (Aim 3). Our design has a number of advantages for this study including 1) it will assess SAIA-HTN's performance under pragmatic conditions, 2) a randomized design reduces potential confounding and will be able to provide robust evidence of SAIA-HTN's impact, and 3) it facilitates assessment of maturation patterns in SAIA-HTN's effect over time, during both the intensive and sustainment phases.

\section{Methods}

\section{Description of the SAIA-HTN implementation strategy}

SAIA-HTN packages systems engineering tools into an iterative, five-step process applied at the facility level to give clinic staff and managers a system-wide view of their cascade performance, identify priority 
areas for improvement, discern modifiable opportunities for improvement, and test these workflow modifications (Figure 1). SAIA has been previously described in the literature.[40, 41] In brief, in its adapted form, the SAIA-HTN cascade includes the following steps:

Step 1: Understand targeted cascade performance, and identify_priority areas for improvement. The HTN cascade analysis tool (HCAT) (Figure 2) uses routine data to provide a rapid, systems-level view of dropoffs along the hypertension cascade for PLHIV, with an optimization function that allows the user to rapidly assess how many additional people will be served if only one step is fully optimized while other stay the same.[28] As an analytic tool, HCAT helps frontline staff and facility managers to prioritize where to intervene by providing a view of the greatest potential for flow improvements across the entire cascade.

Step 2: Process mapping to identify facility-level modifiable bottlenecks. Enabling facility-level staff to identify and gain consensus on bottlenecks to address in their hypertension system is essential to defining innovations to implement. SAIA-HTN applies sequential process flow mapping procedures [35, 42], coupled with workflow observation, to identify bottlenecks and guide discussion on opportunities for workflow modifications.

Step 3: Define and implement facility-specific workflow adaptations to address modifiable bottlenecks. After identifying modifiable barriers within cascade steps, facility staff identify a simple, specific change to improve performance within the targeted step. Selected workflow adaptations should be feasible to implement, be within the scope of influence of facility management and frontline staff, and be expected to lead to rapid, substantial improvements in the targeted cascade step. Ideas for adaptations come from brainstorming solutions with facility staff, complemented by best practices from the literature and high performing services in Mozambique. An implementation plan for the innovation is described in writing by facility and study personnel to ensure consensus among facility staff, and clarify operational design and roles. Steps 3 and 4 are analogous to continuous quality improvement.

Step 4: Monitor changes in routine performance. Facility staff monitor change in routinely reported data from the cascade step selected for improvement. Measuring the absolute change in the proportion of patients progressing through targeted steps captures large, rapid improvements accompanying modifications.

Step 5: Repeat cycle. Systems engineering improvement processes are by definition iterative, with ongoing testing of innovations responsive to evolving, contextually specific barriers. Facility staff repeat steps 1-5 at the end of each cycle to identify new approaches to modify previously identified barriers, or if the first cycle was successful, focusing on improving priority bottlenecks identified in a repeated systems analysis.

\section{SAIA-HTN trial design}


Using a three-year parallel hybrid type III cluster randomized controlled design, we will prospectively implement SAIA-HTN and evaluate its impact for PLHIV individuals in eight intervention and eight control facilities in central Mozambique (CONSORT Checklist, Additional file 1). Public sector district HIV and Non-Communicable Disease (NCD) supervisors, supported by study nurses, will deliver the intervention over a two-year intensive phase, followed by a one-year sustainment phase led by the district HIV and NCD supervisors without additional study personnel support, which will provide evidence on intervention impact under ideal circumstances, as well as the longevity of the effect over time (Table 1). The mixedmethods evaluation will evaluate the impact of SAIA-HTN on clinical as well as process outcomes (Table 2). The ORIC will be applied to facilities earlier in SAIA-HTN implementation (within 3 months of starting) to identify organizational-level attributes that affect intervention adoption. The CFIR framework will be used to guide data collection and interpretation related to implementation, to assess fidelity to intervention protocol, describe intervention adaptations when integrated into routine management systems[43], and identify organizational-level determinants of successful SAIA-HTN implementation. In addition, recurrent measurement of structural readiness and implementation dose (as a function of quality and quantity) at the facility level will inform guidance on essential structural needs to implement SAIA-HTN. Based on trial results, we will model the costs and benefits on hypertension control and HIV viral suppression given different scale-up scenarios within Mozambique. The trial will culminate in a dissemination package, summarizing trial results and providing implementation and cost guidance to support national SAIA-HTN scale-up.

\section{Process for introducing SAIA}

SAIA-HTN's standard operating procedures (SOPs), including the delivery and training schedules as well as the intervention guides and tools (HCAT, process mapping, and CQI guides) were developed during the initial SAIA-HTN pilot and will be refined in preparation for the SAIA-HTN trial. In the three months prior to initiation of the intensive phase, facility teams (including managers and staff from ambulatory and pharmacy services) receive a five-day orientation from study teams and district health authorities on using the SAIA-HTN SOPs, including an introduction to the SAIA tools, the implementation schedule and the data collection procedures. During this first week of SAIA-HTN implementation, facility teams also populate and interpret the HCAT, develop process maps of current PLHIV patient care pathways to hypertension diagnosis and management, define micro-interventions, and indicators to monitor these modifications.

Subsequently, district non-communicable disease (NCD) and HIV supervisors, together with study nurses, introduce SAIA-HTN to intervention health facilities over a two-day period. Within each district, SAIA-HTN is introduced one facility at a time, until all intervention facilities are covered in each district. Facility teams receive bi-weekly supervision visits by study personnel and district supervisors for the first month, followed by monthly visits throughout the remainder of the 24-month intensive implementation period. During the third year after SAIA-HTN introduction (sustainment), monthly mentorship visits will be 
conducted by district authorities without support from study personnel to evaluate SAIA-HTN sustainability with moderate resource investment (including transport to facilities and per diem for staff).

Based on the SAIA trial and SAIA-HTN pilot, it is expected that analysis and improvement cycles will occur monthly, with an average of 12 cycles per year per facility. A SAIA core component that will be maintained in SAIA-HTN is provision to intervention facilities of a flexible facility support fund to address basic equipment needs for hypertension management (sphygmomanometer, scales, stethoscope, etc.), as well as to support workflow modifications, which will continue throughout both the intervention and sustainment periods.

\section{Study setting and eligibility criteria}

\section{Study setting}

Manica and Sofala provinces (population 4 million; Figure 3), have higher adult HIV prevalence than the national average (15.3\% and $15.5 \%$ respectively, compared to $11.5 \%$ nationally).[44] These provinces were selected because of the deep relationship between investigators and health authorities, and absence of structural interventions for hypertension diagnosis and management within HIV care. In central Mozambique, over $98 \%$ of formal health services are offered through the public sector,[45] and primary care utilization is high, supporting spread and likelihood of population-level impact for a supply-side intervention delivered through the HIV treatment platform.

HIV treatment services have been scaled up in most sub-Saharan countries, including Mozambique, where over 1,300 health facilities ( $81 \%$ of public sector health facilities in the country) offer chronic HIV treatment in adult ambulatory services.[46] In this total population of 28 million, more than 850,000 patients are accessing ART.[47, 48] In 2007, Mozambique integrated adult HIV care into general ambulatory services to improve efficiency, reduce stigma, and leverage HIV investments to strengthen the health system. Integrating hypertension screening and management in the HIV care system presents an opportunity to reach a large number of at-risk individuals enrolled in chronic care, and provide a scalable model for application in health centers nationwide.

\section{Eligibility criteria}

To maximize the potential impact of SAIA-HTN, and reflecting the higher burden of hypertension in high volume health facilities, we will select facilities in either provincial or district capitals offering HIV treatment services through ambulatory clinics (Figure 3). Eligible facilities will be public sector clinics, see a minimum of 2,000 total ambulatory consults per month, and be located in central Mozambique. Facilities in which ongoing prospective studies or similar systems analysis and enhancement techniques are being implemented will be excluded from the trial.

\section{Randomization}


Given these criteria, 29 facilities in Manica and Sofala provinces meet eligibility. From these 29, 16 study facilities will be randomly selected (representing 18 of 25 districts in the two provinces) by the study team. Clinics will then be randomly assigned 1:1 to intervention or control using constrained randomization to balance province, urban/ peri-urban, total number of PLHIV and providers by arm.[49] All adult PLHIV (>14 years) will contribute outcomes data via medical records and registries in ambulatory care. Clients will not be directly enrolled as participants in this study.

\section{SAIA-HTN impact assessment}

Through a mentored process of solution identification and testing, we hypothesize that SAIA-HTN will lead to rapid and sustainable improvements in hypertension service delivery in the eight intervention clinics, assessed via routinely available process indicators and patient-level outcome measures.

\section{Study population}

All PLHIV-adults (>14 years) accessing ambulatory care services at study facilities during the study period, including those diagnosed during the consult as well as those diagnosed prior to presentation at care.

\section{Exposure definition}

Facilities will be considered unexposed prior to the initiation of the SAIA-HTN intervention in their health facility and exposed thereafter. Individuals' exposure to the intervention will be based on the exposure status of the facility in the calendar month in which they enter care (including those newly identified as hypertensive and those already diagnosed and on an anti-hypertensive medication).

\section{Outcomes}

The primary SAIA-HTN study process outcome is hypertension screening amongst PLHIV in ambulatory care services, while the primary clinical outcome is controlled hypertension. Secondary outcomes will include additional quantitative measures that reflect successful progression through steps in the hypertension cascade for PLHIV, as well as HIV-related clinical outcomes (Table 2). Process measures were identified for inclusion as study outcomes because they are sensitive to system-level improvements; represent steps that, if changed, would meaningfully alter patterns of hypertension-related morbidity; and are efficient to collect and readily understood by facility managers and frontline staff. Process measures will be assessed monthly over the study period (including three months of pre-intervention baseline measures, 24 months during the intensive intervention phase, and 12 months during the sustainment phase.

Clinical outcomes were included to determine the effect of SAIA-HTN on meaningful hypertension and HIV control over time, which are more dependent on patient behavior and characteristics and may be less sensitive to systems-level improvements. 


\section{Data sources}

Patient-level hypertension and HIV clinical outcomes will be sourced from existing Ministry of Health patient level forms with slight modifications designed to capture the entire hypertension care cascade for PLHIV from screening to controlled hypertension. Data will be abstracted at least weekly into a study database via a RedCAP questionnaire on tablets by study personnel. As part of routine care, each PLHIV patient is assigned a unique identification number that links across service points and clinics, which will be used to abstract registry data for study outcome measures. The database will generate on-demand reports with monthly indicators to populate the HCAT.

\section{Power and sample size}

Power calculations are based on the primary process outcome of the proportion of ambulatory care visits by PLHIV at which blood pressure is measured, comparing the intervention and control facilities during the final three months of the intensive intervention period. Blood pressure screening was selected as the primary outcome as existing guidelines call for it to be routinized into outpatient services for all patients, and it affects the greatest number of PLHIV. Minimum detectable difference calculations were conducted in Stata 14.2 (StataCorp, College Station, Texas), based on a range of plausible values for endline proportion of visits in the control arm and intracluster correlation coefficient (Table 3). Calculations assumed $a=0.05$ and 1000 visits by PLHIV patients over each three-month period. At the start of the pilot study, less than $10 \%$ of routine care visits in the pilot facilities included blood pressure screening; in control facilities, we anticipate there will be minimal improvement in screening coverage during the study period (<5\%). Given these parameters, eight intervention and eight control facilities, we will have $80 \%$ power to detect an absolute difference in the endline proportion of visits with blood pressure screening between $15 \%$ and $29 \%$, depending on endline proportion in the control facilities and intracluster correlation. Given the $31 \%$ increase observed in the pilot study[38], we believe this estimated effect size is reasonable.

\section{Data analysis}

Impact analysis will include generalized linear mixed modeling comparing study outcomes between intervention and control facilities (including the proportion of routine visits at which blood pressure is measured; treatment initiation among those diagnosed with hypertension; medication pickup; and hypertension control among those diagnosed by arm). Models will account for clustering by health facility and will assess the impact of adjustment for patient-level covariates (e.g. age, sex, new vs. existing HIV diagnosis) as well as facility-level covariates (e.g. patient volume, staffing levels), and time (in months since study initiation). Secondary analyses will test for interaction between arm and time, and between arm and study phase (pre-intervention, 24-month intervention, and 12-month sustainment periods). We will also conduct a controlled, segmented time-series analysis that incorporates monthly facility-level estimates from the entire 36-month study period (segmented into three-month preintervention, 24-month intervention, and 12-month sustainment periods). This secondary analysis will 
allow us to fully use available data to assess intervention impact, address both serial and intra-class correlation, and assess temporal patterns in study outcomes.

\section{Determining drivers of SAIA-HTN intervention implementation heterogeneity}

We will apply four implementation science theories, models and frameworks to examine the implementation process, focusing on clinics as the organizational level.

\section{Organizational Readiness for Implementing Change (ORIC)}

ORIC assesses the extent to which organizational members are psychologically and behaviorally prepared to implement organizational change, which affects decisions to adopt implementation strategies like SAIA-HTN.[50,51] Readiness includes: 1) change commitment, reflecting shared resolve to implement a change, and 2) change efficacy, reflecting a shared belief in the collective capability to implement change. To understand readiness for adopting SAIA-HTN, we will apply the ORIC assessment scale which has been translated, adapted, and implemented to this context as part of the SAIA-SCALE study prior to launching SAIA-HTN.[40] We will apply the ORIC to eight management team members of each intervention district $(n=48)$ and eight health workers per intervention facility $(n=64)$. Analysis will test whether sufficient inter-rater reliability and inter-rater agreement exist to aggregate individual responses to the facility level. If tests do not justify aggregation, we will use a measure of intra-facility variability in readiness rather than a facility-level mean in our analysis. The resulting analysis will provide readiness profiles for each facility as they initiate implementation, which will complement adoption, implementation and effectiveness data in understanding the broad impact of SAIA-HTN.

\section{Facility-level structural readiness assessments}

Standardized readiness assessments, adapted from the SARA tool [52] and used in previous SAIA studies, will be carried out annually in all 16 study facilities to assess structural readiness to deliver hypertension services (staffing levels, attributes and training; availability of essential commodities, equipment and supplies; and infrastructure). Recurrent measurement of structural readiness will inform guidance on essential structural needs to implement SAIA-HTN.

\section{Measuring implementation dose}

We have developed an approach to measure implementation dose adapted from McHugh et al's dimensions of implementation for SAIA-HTN.[53] In this definition of dose, the quantity and quality of both the intervention and participation is considered, recognizing that characteristics beyond quantity may influence effectiveness.[54] This information is captured through both reporting tools and focus group discussions (FGDs) and in-depth interviews (IDIs) (Table 4). Reporting tools will be used to capture the quantity, exposure, reach, duration, and elements of quality of implementation across facilities, using internal program reports, field staff observations, supervision reports, archived facility-level process maps and quality improvement plans adapted from the original SAIA and refined during the SAIA-HTN pilot. FGD/IDIs will be used to collect qualitative dimensions of implementation dose including intensity, scope, 
engagement, and features of quality. We will quantify associations between implementation dose and SAIA-HTN cascade improvements to identify best practices, including facility structural characteristics (e.g. staffing levels and experience, patient volume, facility infrastructure, availability of essential drugs, supplies, equipment, etc.). Bivariate analysis and multivariate models will detect associations, specifically correlation between 1) organizational readiness and dose, and 2) process/clinical outcomes and dose. $[55,56]$

\section{Consolidated Framework for Implementation Research (CFIR)}

We will use the CFIR to guide an in-depth examination of the implementation process, define SAIA-HTN core elements, and describe determinants of success and failure across implementing facilities. During the SAIA-HTN pilot we adapted CFIR tools (http://cfirguide.org/; see Table 5 for constructs of interest) to develop semi-structured topic guides to collect data about select constructs from each of the five CFIR domains, supplemented by targeted elicitation of satisfaction with tools/protocols, intent-to-continue-use, and deviations from the 5-step SAIA protocol. After the completion of the intensive intervention phase (year 3), we will conduct FGDs in each intervention facility with 7-10 clinic staff (sufficient to generate conversation without being too large to become intimidating) $(n=56-80)$ participating in the SAIA-HTN. [57] Facilities will be classified as either high or low performing (identified by facilities' fidelity to the SAIAHTN intervention, defined as the number and frequency of SAIA-HTN cycles conducted, and the consistent use of quantitative data to inform progress).[58] By purposively holding separate FGDs for facilities, and classifying them by high and low implementation fidelity, we intend to uncover salient features of successful implementation.

Additional IDIs will be held with facility $(n=24)$ and district $(n=12)$ managers who participate in the SAIA-HTN trial to collect potentially sensitive information that staff members might be hesitant to share in group settings, such as opinions that lower ranking staff feel uncomfortable sharing with their superiors, or sensitive issues related to staffing that higher ranking staff members feel uncomfortable discussing with lower-ranking team members (e.g. leadership engagement or organizational culture). The IDIs will allow for exploration of the individual experience with disseminating and implementing SAIAHTN, and capture intervention adaptations over time, such as staff attitudes or identification with the organization. All FGD and IDI participants will be purposively selected by the study staff, to ensure balance of representation across service location and roles. To understand the implementation process and adaptation during the sustainment phase, we will repeat an equal number of FGDs and IDIs at the end of the sustainment period (year 4), using the same sampling scheme as described above. In addition, implementation tracking (reporting on training and supervision) will be conducted to quantify the executing construct within the implementation process domain.

FGDs and IDIs will be conducted in Portuguese by an experienced facilitator accompanied by a note-taker (FGDs only), audio-recorded, transcribed verbatim into Portuguese by trained Mozambican staff, and translated into English by staff fluent in both language. First, two coders in a stepwise, iterative process will code the IDI and FGD transcripts, and will conduct content analysis within a deductive framework to 
identify key implementation themes (using selected CFIR constructs, but allowing flexibility for other themes to emerge). Coding will be compared across pairs and differences discussed prior to final coding. Case memos will be written and three analysts will assign ratings for each construct. Using a rating process previously applied to the CFIR, $[36,59]$ ratings will reflect the positive or negative influence (valence) and the strength of each construct. Constructs will be coded as missing too much data (M), not (0), weakly (+1/-1), or strongly (+2/-2) distinguishing low/high performance. Findings will be used to develop recommendations for SAIA-HTN implementation, including its core components, intervention adaptions, and lessons learned.

\section{SAIA-HTN economic evaluation}

We will estimate total incremental and unit costs of integrating hypertension diagnosis and management into HIV care. Pending effectiveness, a cost-utility analysis to measure the cost per additional DALY will be conducted.

\section{Cost estimation}

A payer perspective (e.g. Mozambican Ministry of Health) will be used for the analyses (Table 6). Since implementation of SAIA-HTN is integrated into the healthcare system, we will estimate the incremental costs of introducing SAIA-HTN to the existing system. Activity-based cost menus will identify all start-up and recurrent activities and measure resource use and costs from intervention design through implementation. Personnel time for SAIA-HTN tasks will be estimated based on activity tracking and surveys administered to study nurses and district supervisors. We will supplement these data with information from the SAIA-HTN expense reports for human resources, training, supplies, intervention delivery, and facility support for workflow modifications, etc. Cost metrics will include the total incremental costs of the SAIA-HTN intervention and average unit costs, including cost per PLHIV adult 1) screened for HTN, 2) diagnosed with HTN, 3) initiated on HTN medications, 4) adherent to anti-HTN medications (measured via timely medication pick up), 5) maintained controlled HTN, and 6) with HIV viral load suppressed. We will model potential cost-offsets due to the impact of SAIA-HTN on reduced outpatient visits and hospitalizations within the study, using Mozambique unit cost estimates from the WHOCHOICE database.[60]

\section{Modelling outcomes}

Mathematical models will simulate the SAIA-HTN intervention to dynamically evaluate the populationlevel impact of the intervention. We will parameterize the model using a combination of study data (e.g. clinical utilization and outcomes) and estimates from the literature. Model outcomes will include deaths averted due to cardiovascular and cerebrovascular events, and DALYs averted. We will conduct sensitivity analyses to account for uncertainty in key parameters. We will also model the impact of adhering to regional (PASCAR) guidelines for hypertension management for a national cohort of adults in Mozambique and validate the model using Mozambique's STEPwise Approach to Surveillance (STEPS) survey data on hypertension prevalence [7], as well as any other models as they are identified. 
Cost and outcomes comparing SAIA-HTN with the status quo (obtained from the aforementioned analyses) will be combined to calculate the Incremental Cost Effectiveness Ratio (ICER) defined as the incremental cost per death or disability adjusted life year (DALY) averted. We will use the study effectiveness data to estimate the net deaths averted due to cardiovascular and cerebrovascular events. We will estimate the number of DALYs averted to capture the gap between current and ideal health, using study outcomes and assumptions reflecting the Mozambique context (i.e. life expectancy, average duration of illness, etc).[61] Following WHO guidelines,[62] and to facilitate comparisons with other strategies to guide resource allocation, SAIA-HTN will be considered cost-effective, after situating both the costs and effectiveness within the Mozambican context, which will include consideration of the disease burden and the budget of this setting.[63,64] Analysis will be from the payer perspective, taking into consideration productivity and health system costs, as well as future health system costs averted due to prevention of hypertension in PLHIV.

\section{Trial status}

Preparations for SAIA-HTN initiated in May 2019. Initiation of the SAIA-HTN trial is planned for June 2020.

\section{Discussion}

SAIA-HTN is a pragmatic trial to test a novel, scalable approach to optimize hypertension diagnosis and management in PLHIV. The implementation approach aligns with existing administrative structures, providing a practical tool to integrate into routine functions of district and facility supervisors. SAIA uses systems engineering tools to visualize and quantify interconnected service delivery steps, allowing health workers to prioritize interventions that can move more individuals through critical care steps in their clinic. SAIA is flexible and adaptable to local settings, and empowers health care workers to test their ideas for optimization through a series of "micro-changes". Rather than testing a single strategy that may become irrelevant after policy or technology changes, SAIA has longevity, as the approach is adaptable to the changing service landscape, which increasingly includes co-morbid conditions like hypertension.

Our design includes multiple novel implementation science methods. The CFIR provides a guide for intervention planning, implementation, and by addressing "what works, where and why", identifies local barriers to implementation and contributes to the knowledge base around implementation of interventions across diverse settings.[59] Assessing readiness for change identifies organizational-level determinants of adoption and implementation of interventions. We will use the Organizational Readiness for Implementing Change (ORIC) scale to assess facility readiness for SAIA-HTN adoption.[50, 51] Cost effective analyses rarely assess CVD management strategies among PLHIV, and more research is needed.[65] This study will model long-term outcomes (DALYs) to capture the minimum data set for economic evaluation recently developed for researchers studying HIV/NCD integration.[65] State of the art implementation science methods strengthen our evaluative framework for multi-level, theory-based 
adaptation of interventions, and exploits outcome heterogeneity to assess implementation barriers and facilitators.

\section{List Of Abbreviations}

\begin{tabular}{ll} 
ART & Anti-Retroviral Therapy \\
\hline CFIR & Consolidated Framework for Implementation Research \\
\hline CQI & Continuous Quality Improvement \\
\hline CVD & Cardiovascular Disease \\
\hline DALY & Disability Adjusted Life Years \\
\hline FGD & Focus Group Discussion \\
\hline HCAT & Hypertension Cascade Analysis Tool \\
\hline HIV & Human Immunodeficiency Virus \\
\hline HTN & Hypertension \\
\hline ICER & Incremental Cost Effectiveness Ratio \\
\hline IDI & In-Depth Interview \\
\hline LMIC & Low and Middle Income Countries \\
\hline NCD & Non-Communicable Disease \\
\hline ORIC & Organizational Readiness for Implementing Change \\
\hline PASCAR & Pan-African Society of Cardiology \\
\hline PLHIV & People Living with HIV \\
\hline SAIA & Systems Analysis and Improvement Approach \\
\hline SOP & Standard Operating Procedures \\
\hline SSA & sub-Saharan Africa \\
\hline STEPS & STEPwise Approach to Surveillance \\
\hline
\end{tabular}

\section{Declarations}

\section{Ethics}

This study was approved by the institutional review boards of the Eduardo Mondlane University/Maputo Central Hospital (\#CIBS FM\&HCM/96/2018) and the University of Washington in Seattle, USA (\#STUDY00006694). 
Not applicable.

\section{Availability of data and material}

Not applicable.

\section{Competing interests}

The authors declare that they have no competing interests.

\section{Funding}

The Research reported in this publication is supported by NHLBI, NICHD, NIAID, NCI, NIMH, NIDA, NIA of the National Institutes of Health under award numbers R01HL142412 and P30AI027757. The content is solely the responsibility of the authors and does not necessarily represent the official views of the National Institutes of Health.

\section{Authors contributions}

SG, AOM, KA, JC, LA, BC, HC, LH, RH, SH, El, CL, FM, NM, MN, JP, IR, KR, NS, OU, AW, BW, and KS conceived of the study. All authors contributed to refining the study design and finalizing the protocol. SG drafted the final version of the paper. All authors read and approved the final manuscript.

\section{Acknowledgements}

We would like to acknowledge the provincial health departments of Manica and Sofala provinces, Mozambique, for their collaboration in designing and conducting this study.

\section{References}

1. Organization WH: Global Health Risks: Mortality and Burden of Disease Attributable to Selected Major Risks. In. Geneva: World Health Organization; 2009.

2. Ataklte F, Erqou S, Kaptoge S, Taye B, Echouffo-Tcheugui JB, Kengne AP: Burden of undiagnosed hypertension in sub-saharan Africa: a systematic review and meta-analysis. Hypertension 2015, 65(2):291-298.

3. Lim SS, Vos T, Flaxman AD, Danaei G, Shibuya K, Adair-Rohani H, Amann M, Anderson HR, Andrews $\mathrm{KG}$, Aryee $\mathrm{M}$ et al: A comparative risk assessment of burden of disease and injury attributable to 67 risk factors and risk factor clusters in 21 regions, 1990-2010: a systematic analysis for the Global Burden of Disease Study 2010. Lancet 2012, 380(9859):2224-2260.

4. Twagirumukiza M, De Bacquer D, Kips JG, de Backer G, Stichele RV, Van Bortel LM: Current and projected prevalence of arterial hypertension in sub-Saharan Africa by sex, age and habitat: an 
estimate from population studies. J Hypertens 2011, 29(7):1243-1252.

5. World Health Organization: Global Health Estimates 2014 summary tables: deaths by cause, age and sex by WHO region 2000-2012. In. Geneva: World Health Organization; 2014.

6. Ministerio da Saude. In.; 2014.

7. Jessen N, Damasceno A, Silva-Matos C, Tuzine E, Madede T, Mahoque R, Padrao P, Mbofana F, Polonia J, Lunet N: Hypertension in Mozambique: trends between 2005 and 2015. J Hypertens 2018, 36(4):779-784.

8. Antonello VS, Antonello IC, Grossmann TK, Tovo CV, Pupo BB, Winckler Lde Q: Hypertension-an emerging cardiovascular risk factor in HIV infection. J Am Soc Hypertens 2015, 9(5):403-407.

9. Lloyd-Sherlock P, Ebrahim S, Grosskurth H: Is hypertension the new HIV epidemic? Int J Epidemiol 2014, 43(1):8-10.

10. Medina-Torne S, Ganesan A, Barahona I, Crum-Cianflone NF: Hypertension is common among HIVinfected persons, but not associated with HAART. J Int Assoc Physicians AIDS Care (Chic) 2012, 11(1):20-25.

11. Schouten J, Wit FW, Stolte IG, Kootstra NA, van der Valk M, Geerlings SE, Prins M, Reiss P, Group AGCS: Cross-sectional comparison of the prevalence of age-associated comorbidities and their risk factors between HIV-infected and uninfected individuals: the AGEhIV cohort study. Clin Infect Dis 2014, 59(12):1787-1797.

12. Marcus JL, Chao CR, Leyden WA, Xu L, Quesenberry CP, Jr., Klein DB, Towner WJ, Horberg MA, Silverberg MJ: Narrowing the Gap in Life Expectancy Between HIV-Infected and HIV-Uninfected Individuals With Access to Care. J Acquir Immune Defic Syndr 2016, 73(1):39-46.

13. Nguyen KA, Peer N, Mills EJ, Kengne AP: Burden, Determinants, and Pharmacological Management of Hypertension in HIV-Positive Patients and Populations: A Systematic Narrative Review. AIDS ReV 2015, 17(2):83-95.

14. Magodoro IM, Esterhuizen TM, Chivese T: A cross-sectional, facility based study of comorbid noncommunicable diseases among adults living with HIV infection in Zimbabwe. BMC Res Notes 2016, 9:379.

15. Krauskopf K, Van Natta ML, Danis RP, Gangaputra S, Ackatz L, Addessi A, Federman AD, Branch AD, Meinert CL, Jabs DA et al: Correlates of hypertension in patients with AIDS in the era of highly active antiretroviral therapy. J Int Assoc Provid AIDS Care 2013, 12(5):325-333.

16. Xu Y, Chen X, Wang K: Global prevalence of hypertension among people living with HIV: a systematic review and meta-analysis. J Am Soc Hypertens 2017.

17. Friis-Moller N, Weber R, Reiss P, Thiebaut R, Kirk O, d'Arminio Monforte A, Pradier C, Morfeldt L, Mateu $\mathrm{S}$, Law $\mathrm{M}$ et al: Cardiovascular disease risk factors in HIV patients-association with antiretroviral therapy. Results from the DAD study. AIDS 2003, 17(8):1179-1193.

18. Jin C, Ji S, Xie T, Hoxtermann S, Fuchs W, Lu X, Wu H, Cheng L, Skaletz-Rorowski A, Brockmeyer NH et al: Severe dyslipidemia and immune activation in HIV patients with dysglycemia. HIV Clin Trials 2016, 17(5):189-196. 
19. Osegbe ID, Soriyan OO, Ogbenna AA, Okpara HC, Azinge EC: Risk factors and assessment for cardiovascular disease among HIV-positive patients attending a Nigerian tertiary hospital. Pan Afr Med J 2016, 23:206.

20. Ekoru K, Young EH, Dillon DG, Gurdasani D, Stehouwer N, Faurholt-Jepsen D, Levitt NS, Crowther NJ, Nyirenda M, Njelekela MA et al: HIV treatment is associated with a two-fold higher probability of raised triglycerides: Pooled Analyses in 21023 individuals in sub-Saharan Africa. Glob Health Epidemiol Genom 2018, 3.

21. Deeks SG, Lewin SR, Havlir DV: The end of AIDS: HIV infection as a chronic disease. Lancet 2013, 382(9903):1525-1533.

22. Neuhaus J, Jacobs DR, Jr., Baker JV, Calmy A, Duprez D, La Rosa A, Kuller LH, Pett SL, Ristola M, Ross MJ et al: Markers of inflammation, coagulation, and renal function are elevated in adults with HIV infection. $J$ Infect Dis 2010, 201(12):1788-1795.

23. Duprez DA, Neuhaus J, Kuller LH, Tracy R, Belloso W, De Wit S, Drummond F, Lane HC, Ledergerber B, Lundgren $\mathrm{J}$ et al: Inflammation, coagulation and cardiovascular disease in HIV-infected individuals. PLoS One 2012, 7(9):e44454.

24. Kuller LH, Tracy R, Belloso W, De Wit S, Drummond F, Lane HC, Ledergerber B, Lundgren J, Neuhaus J, Nixon $\mathrm{D}$ et al: Inflammatory and coagulation biomarkers and mortality in patients with HIV infection. PLoS Med 2008, 5(10):e203.

25. Dzudie A, Rayner B, Ojji D, Schutte AE, Twagirumukiza M, Damasceno A, Ba SA, Kane A, Kramoh E, Anzouan Kacou JB et al: Roadmap to Achieve 25\% Hypertension Control in Africa by 2025. Glob Heart 2018, 13(1):45-59.

26. Kayima J, Wanyenze RK, Katamba A, Leontsini E, Nuwaha F: Hypertension awareness, treatment and control in Africa: a systematic review. BMC Cardiovasc Disord 2013, 13:54.

27. Guwatudde D, Nankya-Mutyoba J, Kalyesubula R, Laurence C, Adebamowo C, Ajayi I, Bajunirwe F, Njelekela M, Chiwanga FS, Reid T et al: The burden of hypertension in sub-Saharan Africa: a fourcountry cross sectional study. BMC Public Health 2015, 15:1211.

28. Wagner AD, Gimbel S, Asbjornsdottir KH, Cherutich P, Coutinho J, Crocker J, Cruz E, Cuembelo F, Cumbe V, Eastment $\mathrm{M}$ et al: Cascade Analysis: An Adaptable Implementation Strategy Across HIV and Non-HIV Delivery Platforms. J Acquir Immune Defic Syndr 2019, 82 Suppl 3:S322-S331.

29. Baekken M, Os I, Sandvik L, Oektedalen O: Hypertension in an urban HIV-positive population compared with the general population: influence of combination antiretroviral therapy. $J$ Hypertens 2008, 26(11):2126-2133.

30. Sherer R, Solomon S, Schechter M, Nachega JB, Rockstroh J, Zuniga JM: HIV provider-patient communication regarding cardiovascular risk: results from the AIDS Treatment for Life International Survey. J Int Assoc Provid AIDS Care 2014, 13(4):342-345.

31. Lichtenstein KA, Armon C, Buchacz K, Chmiel JS, Buckner K, Tedaldi E, Wood K, Holmberg SD, Brooks JT, Investigators H: Provider compliance with guidelines for management of cardiovascular risk in HIV-infected patients. Prev Chronic Dis 2013, 10:E10. 
32. Ameh S, Klipstein-Grobusch K, Musenge E, Kahn K, Tollman S, Gomez-Olive FX: Effectiveness of an Integrated Approach to HIV and Hypertension Care in Rural South Africa: Controlled Interrupted TimeSeries Analysis. J Acquir Immune Defic Syndr 2017, 75(4):472-479.

33. Rabkin M, Kruk ME, El-Sadr WM: HIV, aging and continuity care: strengthening health systems to support services for noncommunicable diseases in low-income countries. AIDS 2012, 26 Suppl 1:S77-83.

34. Mussa AH, Pfeiffer J, Gloyd SS, Sherr K: Vertical funding, non-governmental organizations, and health system strengthening: perspectives of public sector health workers in Mozambique. Hum Resour Health 2013, 11:26.

35. Wagner AD, Crocker J, Liu S, Cherutich P, Gimbel S, Fernandes Q, Mugambi M, Asbjornsdottir K, Masyuko S, Wagenaar BH et al: Making Smarter Decisions Faster: Systems Engineering to Improve the Global Public Health Response to HIV. Curr HIV/AIDS Rep 2019, 16(4):279-291.

36. Gimbel S, Rustagi AS, Robinson J, Kouyate S, Coutinho J, Nduati R, Pfeiffer J, Gloyd S, Sherr K, Granato SA et al: Evaluation of a Systems Analysis and Improvement Approach to Optimize Prevention of Mother-To-Child Transmission of HIV Using the Consolidated Framework for Implementation Research. J Acquir Immune Defic Syndr 2016, 72 Suppl 2:S108-116.

37. Rustagi AS, Gimbel S, Nduati R, Cuembelo Mde F, Wasserheit JN, Farquhar C, Gloyd S, Sherr K, with input from the SST: Implementation and Operational Research: Impact of a Systems Engineering Intervention on PMTCT Service Delivery in Cote d'Ivoire, Kenya, Mozambique: A Cluster Randomized Trial. J Acquir Immune Defic Syndr 2016, 72(3):e68-76.

38. Gimbel S, Coutinho, J, Sherr K.: Get with the Flow: Optimizing Hypertension Care Cascades for People Living with HIV in Mozambique. In: University of Washington Center for Global Health Nursing Lecture series. Seattle, USA: University of Washington; 2019.

39. Curran GM, Bauer M, Mittman B, Pyne JM, Stetler C: Effectiveness-implementation hybrid designs: combining elements of clinical effectiveness and implementation research to enhance public health impact. Med Care 2012, 50(3):217-226.

40. Sherr K, Asbjornsdottir K, Crocker J, Coutinho J, de Fatima Cuembelo M, Tavede E, Manaca N, Ronen $\mathrm{K}$, Murgorgo F, Barnabas R et al: Scaling-up the Systems Analysis and Improvement Approach for prevention of mother-to-child HIV transmission in Mozambique (SAIA-SCALE): a stepped-wedge cluster randomized trial. Implement Sci 2019, 14(1):41.

41. Sherr K, Gimbel S, Rustagi A, Nduati R, Cuembelo F, Farquhar C, Wasserheit J, Gloyd S, With input from the SST: Systems analysis and improvement to optimize pMTCT (SAIA): a cluster randomized trial. Implement Sci 2014, 9:55.

42. Colligan L, Anderson JE, Potts HW, Berman J: Does the process map influence the outcome of quality improvement work? A comparison of a sequential flow diagram and a hierarchical task analysis diagram. BMC Health Serv Res 2010, 10:7.

43. Wiltsey Stirman S, Baumann AA, Miller CJ: The FRAME: an expanded framework for reporting adaptations and modifications to evidence-based interventions. Implement Sci 2019, 14(1):58. 
44. Ministry of Health: National HIV/AIDS prevalence, behavioral risk and information survey (INSIDA). In. Maputo, Mozambique; 2009.

45. Sherr K, Cuembelo F, Michel C, Gimbel S, Micek M, Kariaganis M, Pio A, Manuel JL, Pfeiffer J, Gloyd S: Strengthening integrated primary health care in Sofala, Mozambique. BMC Health Serv Res 2013, 13 Suppl 2:S4.

46. Ministerio da Saude: Relatorio Anual 2017 HIV. In. Maputo, Mocambique: Ministerio da Saude; 2014.

47. UNAIDS: Country fact sheets Mozambique 2016 - HIV/AIDS. . In.; 2016.

48. World Bank: Data Mozambique. In.; 2018.

49. Moulton LH: Covariate-based constrained randomization of group-randomized trials. Clin Trials 2004, 1(3):297-305.

50. Weiner BJ: A theory of organizational readiness for change. Implement Sci 2009, 4:67.

51. Shea CM, Jacobs SR, Esserman DA, Bruce K, Weiner BJ: Organizational readiness for implementing change: a psychometric assessment of a new measure. Implement Sci 2014, 9:7.

52. Health Statistics and Information Systems: Service Availability and Readiness Assessment (SARA): an annual monitoring system for service delivery, Version 2.2. In. Geneva: World Health Organization; 2015.

53. McHugh M, Harvey JB, Kang R, Shi Y, Scanlon DP: Measuring the Dose of Quality Improvement Initiatives. Med Care Res Rev 2016, 73(2):227-246.

54. O'Neill SM: How do quality improvement interventions succeed? Archetypes of success and failure. . Santa Monica, CA: RAND; 2011.

55. Rustagi AS, Gimbel S, Nduati R, Cuembelo MdF, Wasserheit JN, Farquhar C, Gloyd S, Sherr K: Health facility factors and quality of services to prevent mother-to-child HIV transmission in Côte d'Ivoire, Kenya, and Mozambique. International Journal of STD \& AIDS, 0(0):0956462416668766.

56. Wagenaar BH, Gimbel S, Hoek R, Pfeiffer J, Michel C, Manuel JL, Cuembelo F, Quembo T, Afonso P, Gloyd $S$ et al: Stock-outs of essential health products in Mozambique - longitudinal analyses from 2011 to 2013. Trop Med Int Health 2014, 19(7):791-801.

57. Krueger R, Casey MA: Focus groups: a practical guide for applied research: SAGE Publications; 2000.

58. Taylor MJ, McNicholas C, Nicolay C, Darzi A, Bell D, Reed JE: Systematic review of the application of the plan-do-study-act method to improve quality in healthcare. BMJ Qual Saf 2014, 23(4):290-298.

59. Damschroder LJ, Aron DC, Keith RE, Kirsh SR, Alexander JA, Lowery JC: Fostering implementation of health services research findings into practice: a consolidated framework for advancing implementation science. Implement Sci 2009, 4:50.

60. Choosing Interventions that are Cost-Effective. Estimates of Unit Costs of Patient Services [http://www.who.int/choice/cost-effectiveness/inputs/health_service/en/]

61. Murray CJ, Vos T, Lozano R, Naghavi M, Flaxman AD, Michaud C, Ezzati M, Shibuya K, Salomon JA, Abdalla $S$ et al: Disability-adjusted life years (DALYs) for 291 diseases and injuries in 21 regions, 
1990-2010: a systematic analysis for the Global Burden of Disease Study 2010. Lancet 2012, 380(9859):2197-2223.

62. Commission on Macroeconomics and Health: Macroeconomics and health: investing in health for economic development. In. Boston: Center for International Development at Harvard University 2001.

63. Galarraga O, Colchero MA, Wamai RG, Bertozzi SM: HIV prevention cost-effectiveness: a systematic review. BMC Public Health 2009, 9 Suppl 1:S5.

64. Marseille E, Larson B, Kazi DS, Kahn JG, Rosen S: Thresholds for the costeffectiveness of interventions: alternative approaches. Bull World Health Organ 2015, 93(2):118-124.

65. Nugent R, Barnabas RV, Golovaty I, Osetinsky B, Roberts DA, Bisson C, Courtney L, Patel P, Yonga G, Watkins D: Costs and costeffectiveness of HIV/noncommunicable disease integration in Africa: from theory to practice. AIDS2018, 32 Suppl 1:S83-S92.

66. Drummond MF, Jefferson TO: Guidelines for authors and peer reviewers of economic submissions to the BMJ. The BMJ Economic Evaluation Working Party. BMJ 1996, 313(7052):275-283.

\section{Tables}

Due to technical limitations, full-text HTML conversion of the tables could not be completed. However, the manuscript can be downloaded and accessed as PDF in the Supplementary Files section below.

\section{Figures}




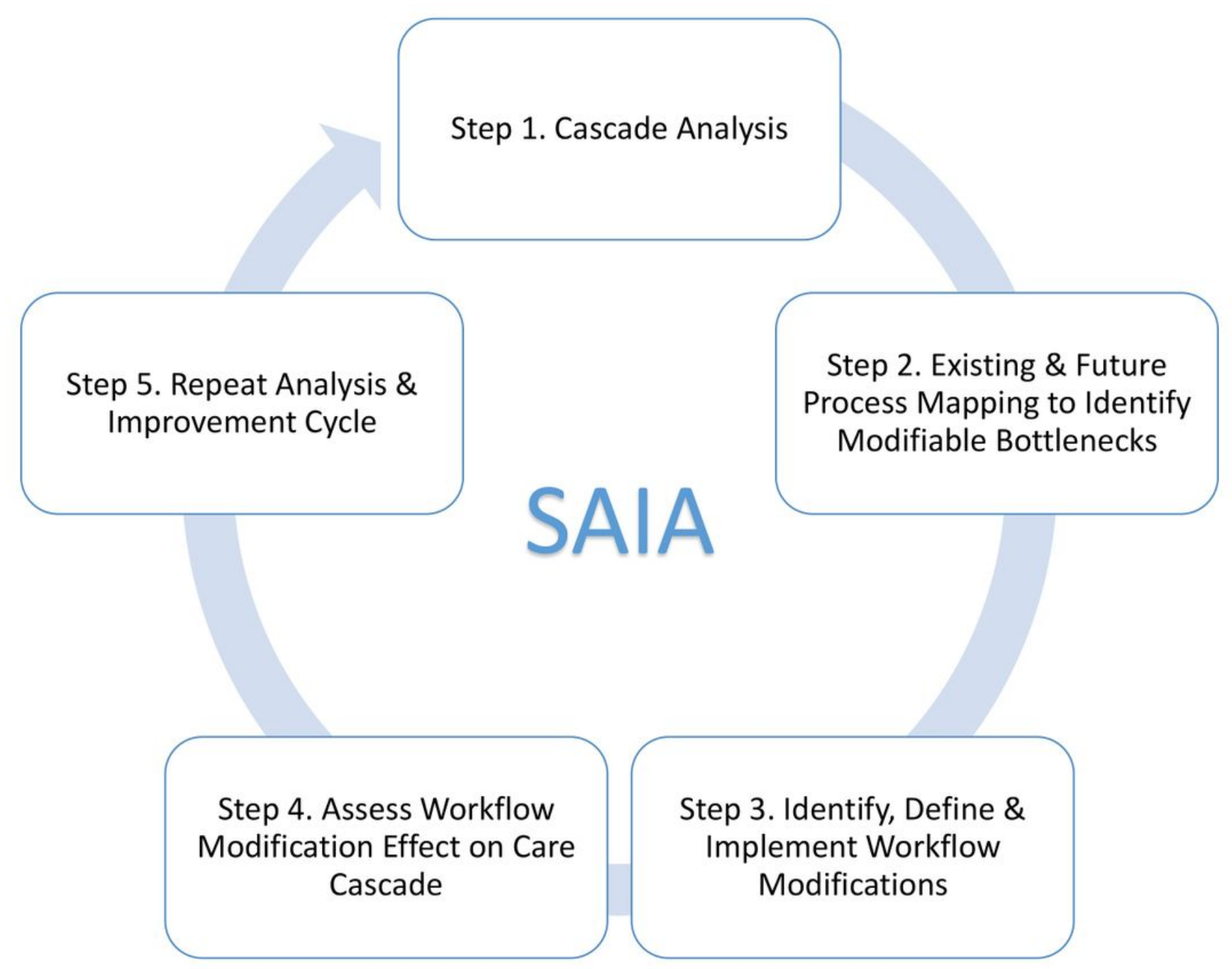

Figure. 1 The Systems Analysis \& Improvement Approach (SAIA)

Figure 1

The Systems Analysis \& Improvement Approach (SAIA) 


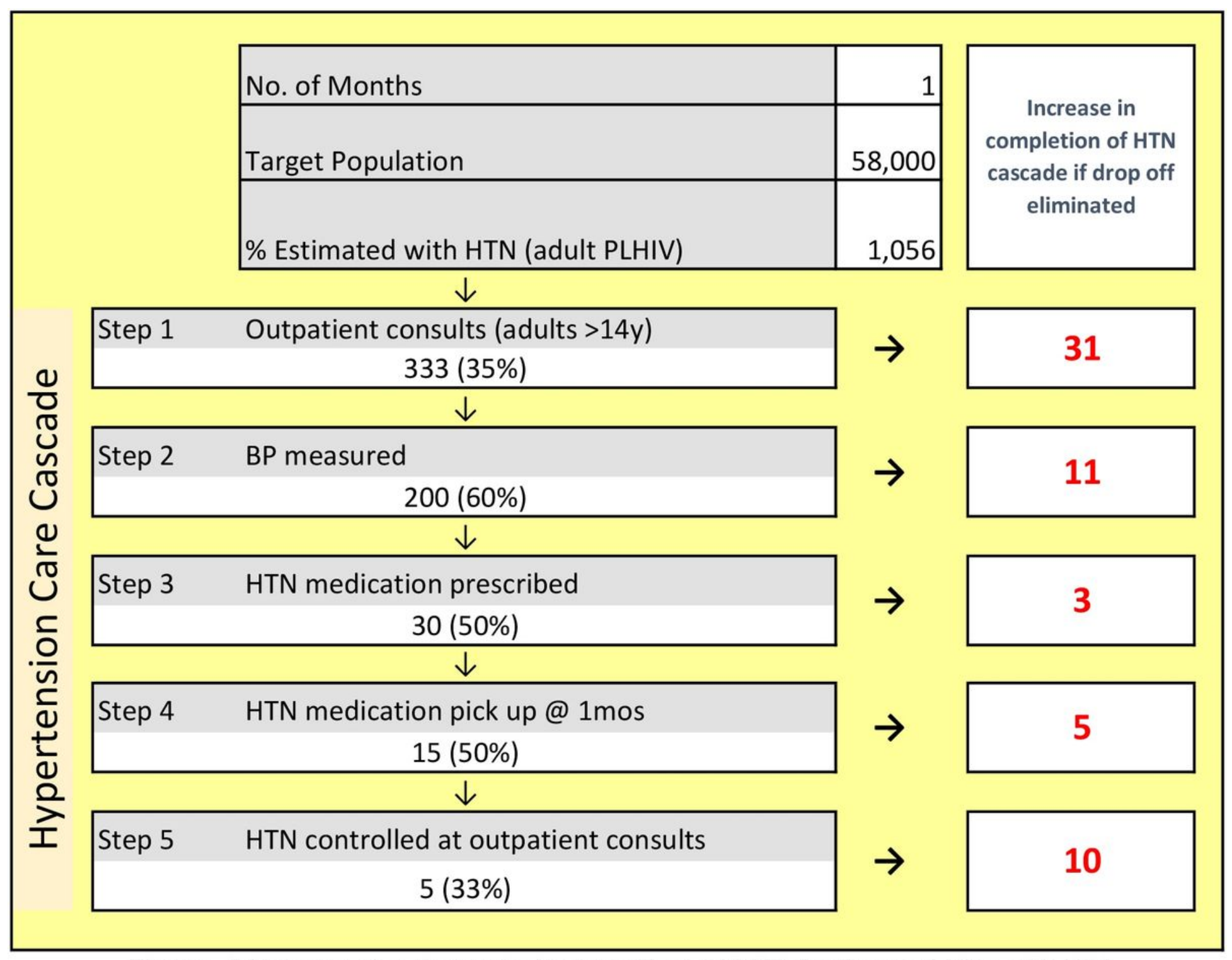

Figure. 2 Hypertension Cascade Analysis Tool (HCAT) for People Living with HIV

Figure 2

Hypertension Cascade Analysis Tool (HCAT) for People Living with HIV 

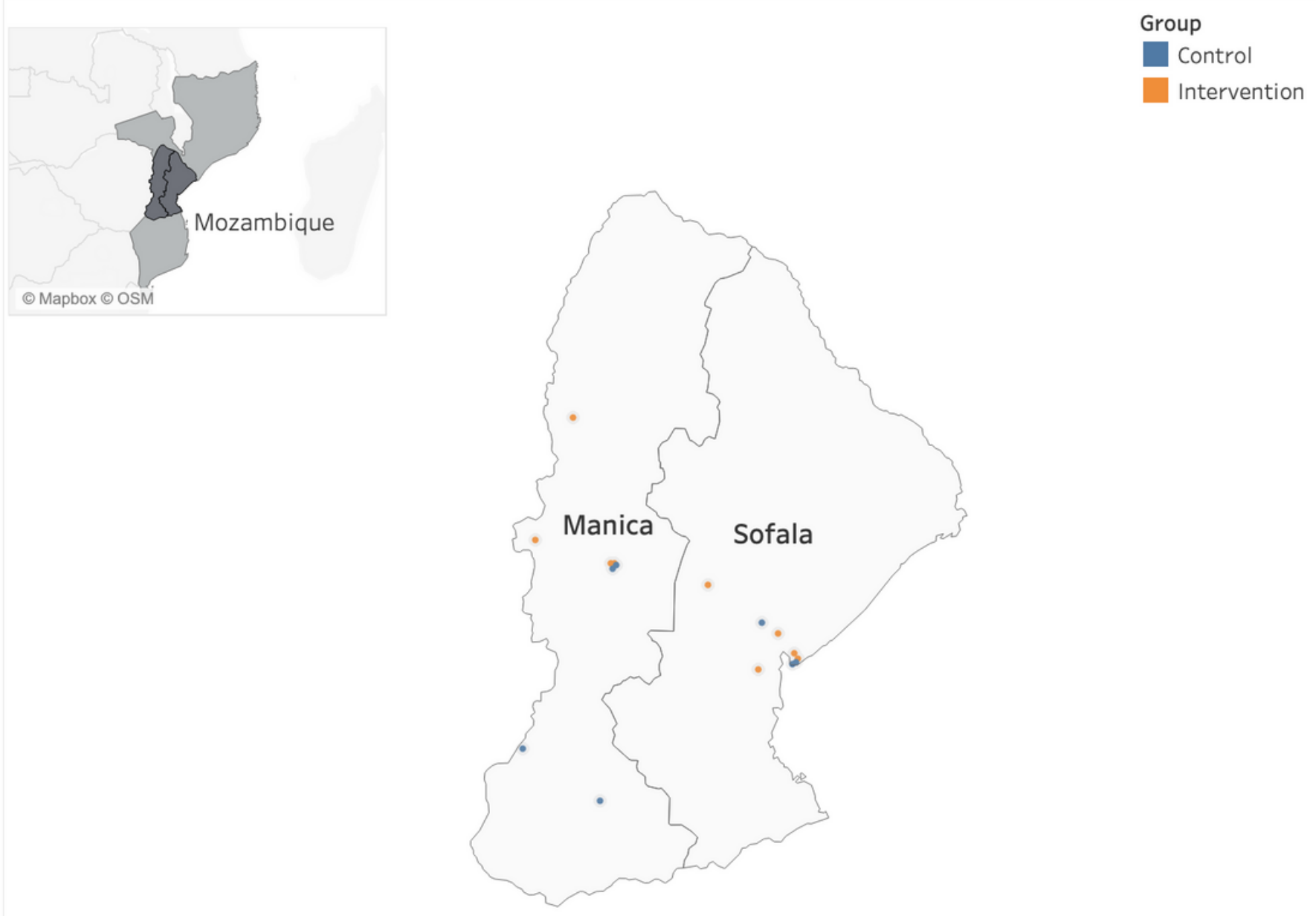

Fig 3. Map of Study Area (Manica and Sofala Provinces, Mozambique)

() 2020 Mapbox @ OpenStreetMap

\section{Figure 3}

Map of Study Area

\section{Supplementary Files}

This is a list of supplementary files associated with this preprint. Click to download.

- Table4.DimensionsReportingof ImplementationDose.pdf

- Table6.SAIAHTNEconomicEvaluationSummary.pdf

- Table1.StudyTimeline.pdf

- Table2.SAIAHTNStudyPrimarySecondaryOutcomes.pdf

- Table3.MinimumDetectableAlternative.pdf

- Table5.CFIRConstructsof Interest.pdf 